\title{
Спектры остаточной фотопроводимости в гетероструктурах с квантовыми ямами $\mathrm{HgTe} / \mathrm{CdHgTe}$
}

\author{
(C) К.Е. Спирин ${ }^{1}$, Д.М. Гапонова ${ }^{1,1}$, В.И. Гавриленко ${ }^{1,2}$, Н.Н. Михайлов ${ }^{3}$, С.А. Дворецкий ${ }^{3}$ \\ ${ }^{1}$ Институт фризики микроструктур Российской академии наук, \\ 603950 Нижний Новгород, Россия \\ ${ }^{2}$ Нижегородский государственный университет им. Н.И. Лобачевского, \\ 603950 Нижний Новгород, Россия \\ ${ }^{3}$ Институт ффизики полупроводников им. А.В. Ржанова Сибирского отделения Российской академии наук, \\ 630090 Новосибирск, Россия \\ ฯ E-mail: dmg@ipmras.ru
}

Поступила в Редакцию 24 апреля 2019 г.

В окончательной редакции 29 апреля 2019 г.

Принята к публикации 29 апреля 2019 г.

Исследованы спектры остаточной фотопроводимости (ОФП) в гетероструктурах с квантовыми ямами $\mathrm{HgTe} / \mathrm{CdHgTe} n$ - и $p$-типа проводимости при температуре $T=4.2 \mathrm{~K}$. Показано, что в зависимости от длины волны подсветки ОФП может быть как положительной (увеличение концентрации носителей в квантовой яме), так и отрицательной, причем максимумам ОФП в образце $n$-типа проводимости в целом соответствуют минимумы ОФП в образцах $p$-типа и наоборот. В образцах $p$-типа обнаружено, что подсветка при определенных длинах волн приводит к „вымораживанию“ свободных носителей в КЯ, но не к конверсии типа проводимости, что указывает на важную роль в механизме ОФП встроенного электрического поля, которое „выключается“ при нейтрализации квантовой ямы.

Ключевые слова: гетероструктура $\mathrm{HgTe} / \mathrm{CdHgTe}$, квантовая яма, остаточная фотопроводимость.

DOI: $10.21883 /$ FTP.2019.10.48297.43

\section{1. Введение}

Интерес к гетероструктурам с квантовыми ямами (КЯ) $\mathrm{HgTe} / \mathrm{CdHgTe}$ связан в первую очередь с возможностью реализации в них топологического фазового перехода. При толщине КЯ больше критической, $d>d_{c} \approx 6.3$ нм, в КЯ происходит инверсия зон и структура переходит в состояние двумерного топологического изолятора $[1,2]$. При изучении зонной структуры КЯ часто возникает необходимость изменения концентрации носителей, что при магнитотранспортных исследованиях обычно реализуется путем приложения напряжения к нанесенному на поверхность структуры металлическому затвору. Для магнитооптических исследований при низких температурах более удобным способом является использование эффекта остаточной, т. е. сохраняющейся после выключения подсветки, фотопроводимости (ОФП) (см., например, [3-5]). В работе [5] мы впервые исследовали спектры биполярной ОФП в структурах с КЯ $\mathrm{HgTe} / \mathrm{CdHgTe}$ и показали, что в зависимости от длины волны подсветки концентрация носителей заряда может изменяться более чем на порядок. В настоящей работе мы показываем кардинальные различия в спектрах ОФП для структур $n$ - и $p$-типа проводимости.

\section{2. Исследуемые образцы и методика эксперимента}

Исследуемые структуры с КЯ были выращены методом молекулярно-лучевой эпитаксии на подложках
$\operatorname{GaAs}(013)$ с метаморфным буферным и покрывающим слоями CdTe, они содержали КЯ НgTe, окруженную широкозонными барьерами $\mathrm{Cd}_{x} \mathrm{Hg}_{1-x}$ Те. Параметры слоев для всех исследуемых образцов представлены в таблице. Структуры 110621-110624 специально не легировались, при гелиевой температуре они имели остаточную темновую проводимость $p$-типа (как и структуры с двойными КЯ $\mathrm{HgTe} / \mathrm{CdHgTe,} \mathrm{исследовавшиеся} \mathrm{в} \mathrm{рабо-}$ те [5]). В структуре 101221 проводилось симметричное селективное легирование барьерных слоев индием, и структура имела остаточную проводимость $n$-типа. Концентрации носителей заряда определялись методом Ван-дер-Пау.

При исследованиях остаточной фотопроводимости образцы размещались в криомагнитной вставке в транспортный гелиевый сосуд Дьюара СТГ-40. На образец квадратной формы размерами $5 \times 5$ мм по краям наносились два полосковых омических контакта. Через образец пропускался постоянный ток 0.1-100 мкА. С помощью гибкого многожильного световода образец освещался монохроматическим излучением с выхода решеточного монохроматора МДР-23 [3]. Измерялось изменение сопротивления образца в зависимости от длины волны падающего излучения. Для образца 101221 методом Вандер-Пау были также измерены значения концентрации электронов при различных длинах волн подсветки.

\section{3. Результаты и обсуждение}

На рис. 1 представлены измеренный спектр ОФП (в единицах сопротивления $R$ ) образца $101221 n$-типа 
Параметры исследованных образцов $\mathrm{HgTe} / \mathrm{CdHgTe}$

\begin{tabular}{c|c|c|c}
\hline $\begin{array}{c}\text { Номер } \\
\text { образца }\end{array}$ & $\begin{array}{c}\text { Содержание } \mathrm{Cd} \text { в барьерном } \\
\text { слое } \mathrm{Cd}_{x} \mathrm{Hg}_{1-x} \mathrm{Te} x\end{array}$ & $\begin{array}{c}\text { Ширина КЯ, } \\
\text { нм }\end{array}$ & $\begin{array}{c}\text { Темновая концентрация } \\
\text { носителей при } T=4.2 \mathrm{~K}\end{array}$ \\
\hline 101221 & 0.41 & 8 & $n \approx 7 \cdot 10^{10} \mathrm{~cm}^{-2}$ \\
110621 & 0.7 & 6.3 & $p \approx 1 \cdot 10^{11} \mathrm{~cm}^{-2}$ \\
110622 & 0.65 & 5.6 & $p \approx 9 \cdot 10^{10} \mathrm{~cm}^{-2}$ \\
110623 & 0.62 & 6 & $p \approx 6 \cdot 10^{10} \mathrm{~cm}^{-2}$ \\
110624 & 0.62 & 7.1 & $p \approx 3.5 \cdot 10^{10} \mathrm{~cm}^{-2}$
\end{tabular}

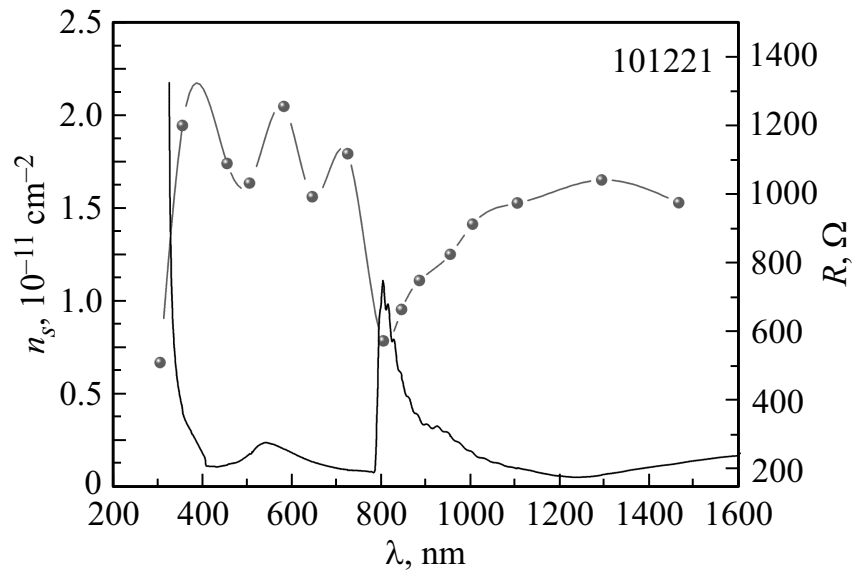

Рис. 1. Спектр остаточной фотопроводимости для образца $\mathrm{HgTe} / \mathrm{CdHgTe} n$-типа проводимости 101221. Точки - значения концентрации электронов в квантовой яме в зависимости от длины волны падающего излучения.

проводимости (сплошная линия) и зависимость концентрации электронов $n_{s}$ от длины волны подсветки $\lambda$ (точки). Спектральная зависимость ОФП качественно повторяет основные особенности спектров образцов, исследованных в работе [5], где подсветка приводила к конверсии типа проводимости с дырочного на электронный. На рис. 2 представлены спектры ОФП 4 исследуемых последовательно выращенных образцов $p$-типа. Как видно из рис. 1, коротковолновая подсветка $(350<\lambda<700 \mathrm{Hм})$ приводит более чем к двукратному росту концентрации электронов по сравнению с темновым значением, что сопровождается еще более сильным падением сопротивления, указывающим на увеличение подвижности носителей заряда, обусловленного, очевидно, экранированием случайного потенциала кулоновских рассеивателей (ср. с [5]). Как и в работе [5], в этой области спектра имеются две особенности: локальный максимум сопротивления (и соответствующий минимум концентрации электронов) на длине волны $\lambda \approx 520-530$ нм и резкий рост сопротивления при $\lambda=780$ нм. Соответствующая первой особенности энергия кванта 2.4 эВ близка к сумме ширины запрещенной зоны в покрывающем слое $\mathrm{CdTe} \approx 1.6$ эВ и энергии спинового расщепления в валентной зоне $\Delta_{S O} \approx 0.91$ эВ [6]. Резонансный характер особенности объясняется фотовозбуждением некоего локализованно- го центра, связанного со спин-отщепленной зоной. На рис. 3 показана схема перераспределения носителей заряда в структуре при подсветке, приводящего к возникновению ОФП, аналогичная представленной в работе [5]. При большой энергии кванта излучения в покрывающем слое CdTe происходит межзонная генерация электроннодырочных пар. Под действием встроенного электрического поля, направленного от подложки в сторону поверхности структуры, происходит пространственное разделение носителей заряда и увлечение электронов к КЯ, увеличивается концентрация носителей в образце, и, как видно из рис. 1, при освещении в области спектра $350<\lambda<700$ нм она более чем в 2 раза превосходит

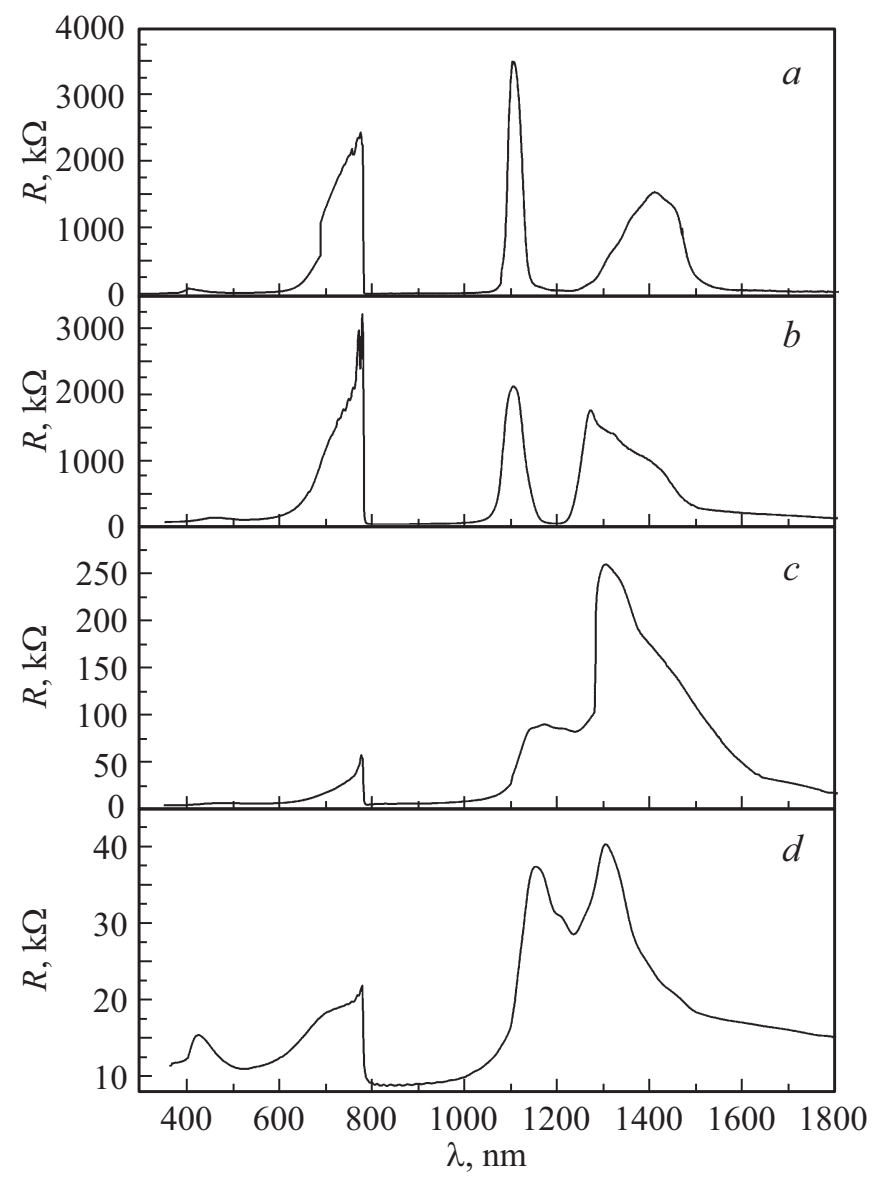

Рис. 2. Спектры остаточной фотопроводимости для исследуемых образцов $\mathrm{HgTe} / \mathrm{CdHgTe} p$-типа проводимости: $a-110621$, $b-110622, c-110623, d-110624$. 


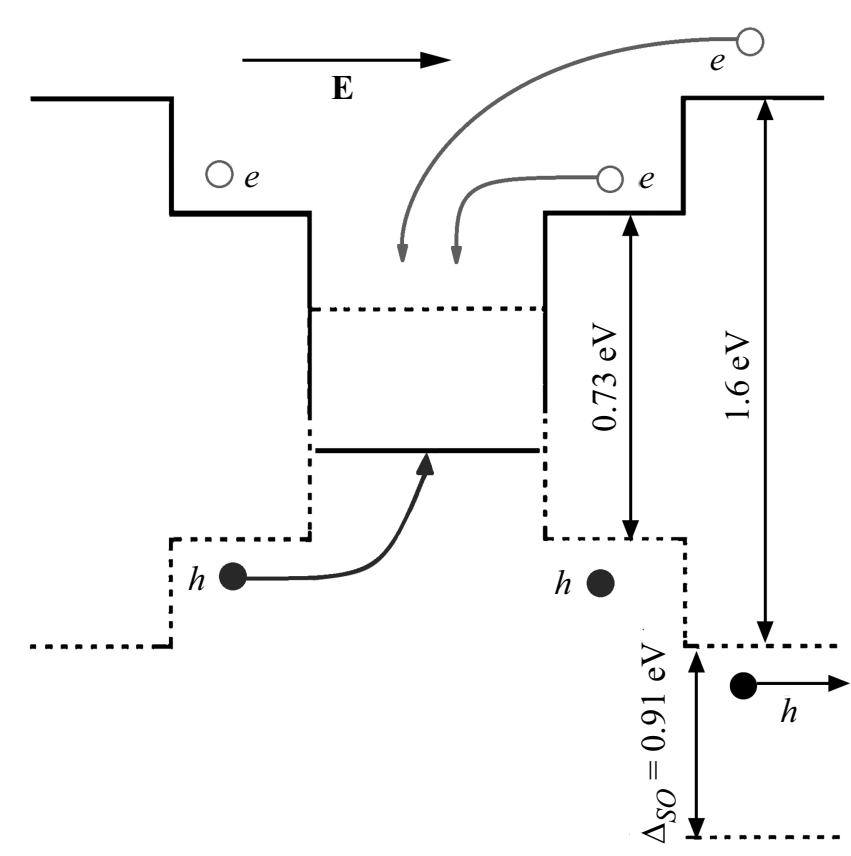

Рис. 3. Схема перераспределения электронов $(e)$ и дырок $(h)$ внутри структуры под действием падающего излучения.

темновую концентрацию. Дырки при этом захватываются на ловушки, в роли которых могут выступать вакансии ртути - двойные акцепторы. Ключевая роль поглощения именно в $\mathrm{CdTe}$, а не в барьерных слоях $\mathrm{CdHgTe,} \mathrm{подтверждается} \mathrm{резким} \mathrm{возрастанием} \mathrm{сопро-}$ тивления и падением электронной концентрации практически до темнового значения при длине волны 780 нм, соответствующей красной границе межзонного поглощения в CdTe. При дальнейшем увеличении длины волны происходит сравнительно плавный рост концентрации электронов в КЯ и соответствующее уменьшение сопротивления, аналогичные наблюдавшимся в работе [5]. В этой области спектра генерация электронно-дырочных пар происходит на межзонных переходах в барьерных слоях $\mathrm{CdHgTe,} \mathrm{непосредственно} \mathrm{примыкающих} \mathrm{к} \mathrm{КЯ,}$ где дырки имеют меньшую вероятность быть захваченными в КЯ, поскольку для этого им пришлось бы двигаться против электрического поля. С ростом длины волны и уменышением кинетической энергии фотовозбужденных дырок вероятность их захвата в КЯ уменьшается, что и обусловливает рост электронной концентрации и падение сопротивления, наблюдаемые вплоть до $\lambda \approx 1300$ нм.

Совершенно иная спектральная картина наблюдается в серии последовательно выращенных нелегированных гетероструктур с КЯ 110621-110624 р-типа проводимости (рис. 2). В отличие от исследованных ранее гетероструктур с двойными КЯ [5] и других нелегированных гетероструктур с одиночными КЯ $\mathrm{HgTe} / \mathrm{CdHgTe}$, в структурах этой серии ни при каких условиях не наблюдается конверсия типа проводимости - при любой длине волны подсветки в структурах сохраняется $p$-тип проводимости. Сопоставляя спектры ОФП на рис. 1 и 2, можно заметить общую тенденцию: максимумам сопротивления на рис. 1 соответствуют минимумы на рис. 2 и наоборот. Так, максимуму сопротивления при $\lambda=520-530$ нм в образцах $n$-типа (рис. 1$)$, связываемому с генерацией высокоэнергетичных дырок при переходах из спин-отщепленной зоны в зону проводимости $\mathrm{CdTe}$ в образцах $p$-типа соответствует минимум сопротивления (рис. 2). В последних локальный максимум сопротивления наблюдается при $\lambda=400-450$ нм (при энергии фотонов 2.8-3.1 эВ), что может быть обусловлено возбуждением электронов в зону проводимости с неких локализованных центров, связанных со спинотщепленной подзоной.

Основными спектральными особенностями на рис. 2 являются три максимума сопротивления, которые наиболее ярко выражены для образцов 110621 и 110622, с максимальными концентрациями дырок в КЯ $\sim 10^{11} \mathrm{~cm}^{-2}$ (рис. 2, $a, b)$. Величина сопротивления в этих максимумах достигает $3 \mathrm{MOM,} \mathrm{что} \mathrm{фактически} \mathrm{означает} \mathrm{пе-}$ реход образца в состояние изолятора. Наиболее коротковолновый из этих максимумов наблюдается при приближении длины волны к значению 780 нм, соответствующему ширине запрещенной зоны в $\mathrm{CdTe}$, после чего наблюдается резкий спад, в отличие от образцов $n$-типа, где при прохождении этой длины волны (в сторону ее увеличения), напротив, наблюдался резкий рост сопротивления (см., например, [5], рис. 1). „Вымораживание“ дырок в КЯ при $\lambda \approx 780$ нм естественно связать с межзонной генерацией электронно-дырочных пар при межзонных переходах в покрывающем слое $\mathrm{CdTe}$. Возникающие электроны увлекаются встроенным электрическим полем в КЯ и компенсируют имеющиеся там дырки. Процесс прекращается при полной компенсации дырок, приводящей к „выключению“ встроенного электрического поля. Падение сопротивления при $\lambda<780$ нм можно объяснить тем, что с ростом энергии кванта фотовозбужденные дырки приобретают все бо́льшую кинетическую энергию, что препятствует их немедленному захвату на ловушки, в результате чего они получают возможность продиффундировать в глубь структуры и быть захваченными в КЯ.

Яркие длинноволновые спектральные особенности $(\lambda>1000$ нм $)$, наблюдаемые в исследуемых образцах $p$-типа проводимости (рис. 2), не коррелируют однозначно с меж(под)зонными расстояниями в исследуемых структурах и связаны, очевидно, с фотовозбуждением локализованных центров, расположенных в валентной зоне в барьерных слоях $\mathrm{Cd}_{x} \mathrm{Hg}_{1-x} \mathrm{Te}$, приводящим к возбуждению электронов в зону проводимости, „сваливающихся“ в КЯ. Подтверждением расположения центров именно в барьерных слоях является несомненная зависимость положения максимумов особенностей от состава барьеров (см. таблицу). С уменьшением доли $\mathrm{Cd}$ в барьерном слое спектральные пики сближаются, в то время как никакой зависимости от ширины КЯ не наблюдается. 


\section{4. Заключение}

Таким образом, нами показано, что в гетероструктуpax $\mathrm{HgTe} / \mathrm{CdHgTe} \mathrm{c} \mathrm{KЯ} \mathrm{наблюдается} \mathrm{сильная} \mathrm{зависи-}$ мость проводимости образца от длины волны падающего излучения, позволяющего значительно перестраивать сопротивление образца, вплоть до превращения образца в изолятор. Наличие такой зависимости позволяет в процессе различных экспериментальных исследований простым методом добиваться перестройки концентрации носителей.

\section{Финансирование работы}

Работа выполнена в рамках государственного задания ИФМ РАН, тема 0035-2019-0020-С01 и при поддержке РФФИ, грант 18-02-00309. В работе использовано оборудование ЦКП ИФМ РАН.

\section{Конфликт интересов}

Авторы заявляют, что у них нет конфликта интересов.

\section{Список литературы}

[1] B.A. Bernevig, T.L. Hughes, S.C. Zhang. Science, 314, 1757 (2006).

[2] M. Konig, S. Wiedmann, C. Brune, A. Roth, H. Buhmann, L.W. Molenkamp, X.-L. Qi, S.-C. Zhang. Science, 318, 766 (2007).

[3] В.Я. Алешкин, В.И. Гавриленко, Д.М. Гапонова, А.В. Иконников, К.В. Маремьянин, С.В. Морозов, Ю.Г. Садофьев, S.R. Johnson, Y.-H. Yang. ФТП, 39, 30 (2005).

[4] А.В. Иконников, А.А. Ластовкин, К.Е. Спирин, М.С. Жолудев, В.В. Румянцев, К.В. Маремьянин, А.В. Антонов, В.Я. Алешкин, В.И. Гавриленко, С.А. Дворецкий, Н.Н. Михайлов, Ю.Г. Садофьев, N. Samal. Письма ЖЭТФ, 92, 837 (2010).

[5] К.Е. Спирин, Д.М. Гапонова, К.В. Маремьянин, В.В. Румянцев, В.И. Гавриленко, Н.Н. Михайлов, С.А. Дворецкий. ФТП, 52, 1482 (2018).

[6] K.H. Yoo, R.L. Aggarwal, L.R. RamMohan, O.K. Wu. J. Vac. Sci. Technol. A, 8, 1194 (1990).

Редактор Л.В. Шаронова

\section{Spectra of persistent photoconductivity in $\mathrm{HgTe} / \mathrm{CdHgTe}$ quantum well heterostructures}

\author{
K.E. Spirin ${ }^{1}$, D.M. Gaponova ${ }^{1}$, V.I. Gavrilenko ${ }^{\mathbf{1 , 2}}$, \\ N.N. Mikhailov ${ }^{3}$, S.A. Dvoretsky \\ ${ }^{1}$ Institute for Physics of Microstructures, \\ Russian Academy of Sciences, \\ 603950 Nizhny Novgorod, Russia \\ ${ }^{2}$ Lobachevsky State University, \\ 603950 Nizhny Novgorod, Russia \\ ${ }^{3}$ Rzhanov Institute of Semiconductor Physics, \\ Siberian Branch of Russian Academy of Sciences, \\ 630090 Novosibirsk, Russia
}

Abstract Spectra of persistent photoconductivity (PPC) in $\mathrm{HgTe} / \mathrm{CdHgTe}$ quantum well $(\mathrm{QW})$ heterostructures with both $n$ and $p$-types conductivity have been investigated at the temperatyre $T=4.2 \mathrm{~K}$. PPC is shown to be either positive (increase of carrier concentration in $\mathrm{QW}$ ) or negative depending on the illumination wavelength. As a general trend, PPC maxima in $n$-type sample correspond to PPC minima in $p$-type samples and vice versa. It is discovered that in $p$-type samples the illumination with certain wavelengths results in freezing out of free carriers in QWs but not in the conversion of the conductivity type. The latter indicates a significant role in the PPC mechanism of the built-in electric field that is switching off at the QW neutralization.

Публикащия материалов Симпозиума завершена. 